Supporting Information

\title{
Real-Time Imaging of Laser-Induced Nanowelding of Silver Nanoparticles in Solution
}

Ariel Rogers ${ }^{1, \dagger}$, Isabelle I. Niyonshuti ${ }^{2, \dagger}$, Alice Cai ${ }^{2}$, Feng Wang ${ }^{2}$, Mourad Benamara ${ }^{3}$, Jingyi Chen ${ }^{2,4, *}$, Yong Wang ${ }^{1,4,5}$,*

${ }^{1}$ Department of Physics, ${ }^{2}$ Department of Chemistry and Biochemistry, ${ }^{3}$ Institute for Nanoscience and Engineering, ${ }^{4}$ Materials Science and Engineering Program, ${ }^{5}$ Cell and Molecular Biology Program, University of Arkansas, Fayetteville, AR 72701.

†These authors contributed equally.

*Corresponding authors: Yong Wang (yongwang@uark.edu) and Jingyi Chen (chenj@uark.edu)

\section{Supplementary Figures}

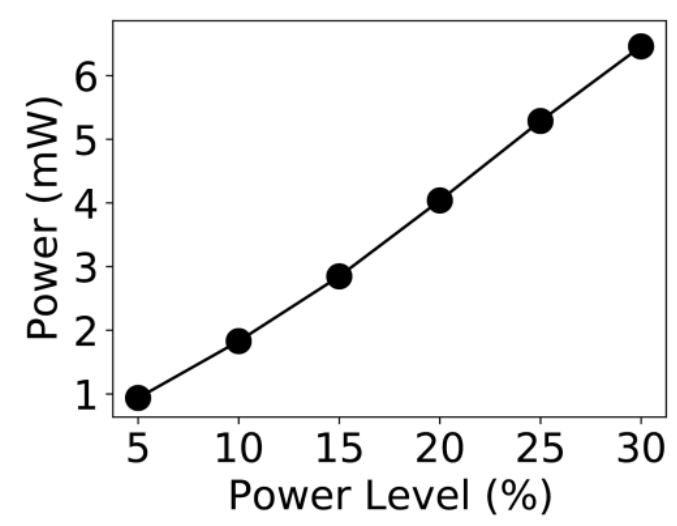

Figure S1. Dependence of the actual laser power $(405 \mathrm{~nm})$ at the samples on the power level in the control panel of the laser. 

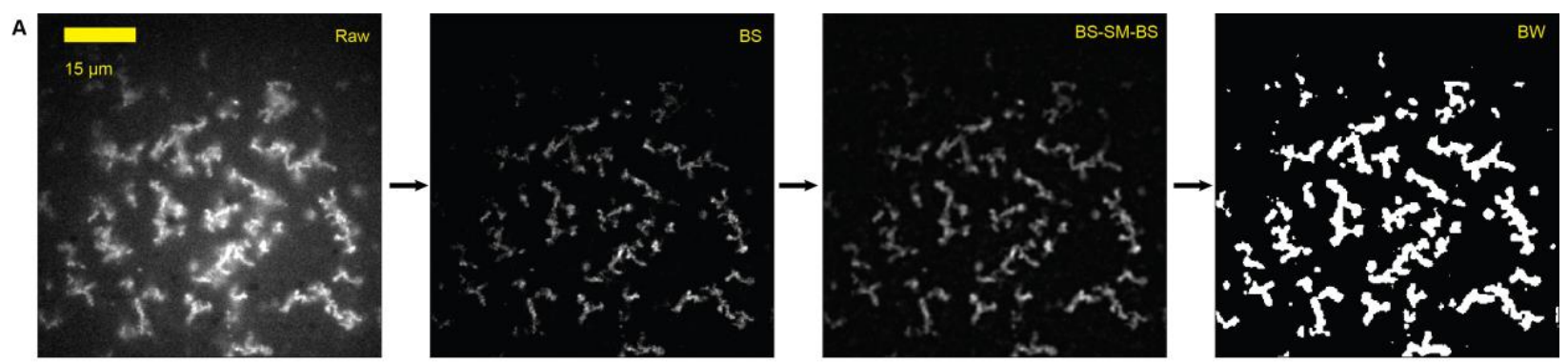

B

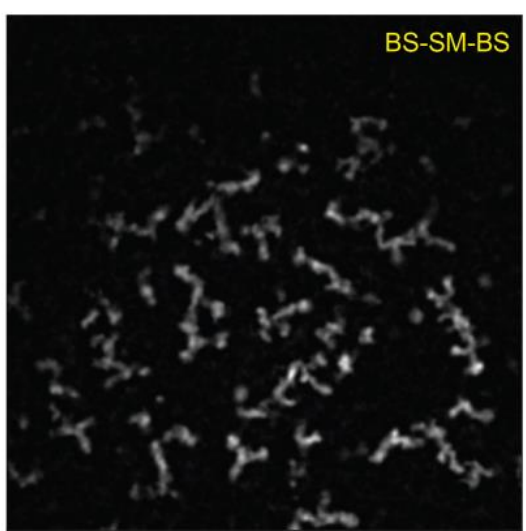

Same Color Scale

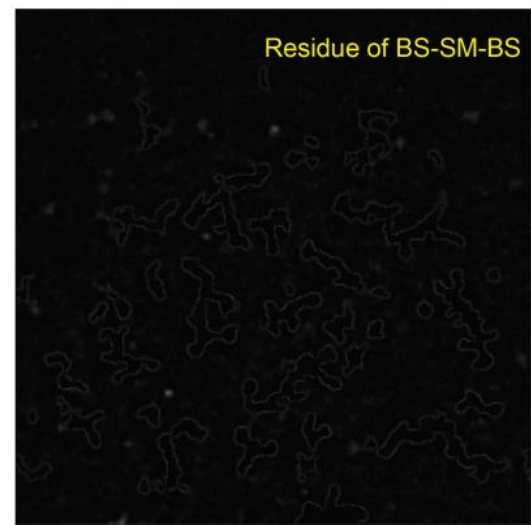

Figure S2. (A) Example of image pre-processing for automatic identification of the laserinduced assemblies. Raw: raw image. BS: after background-subtraction. BS-SM-BS: after background-subtraction, smoothing, and background-subtraction. BW: black/white image after thresholding. (B) Comparison of the pre-processed image of the laser-induced assemblies with the corresponding residual image after automated structure-identification, presented at the same color scale. 


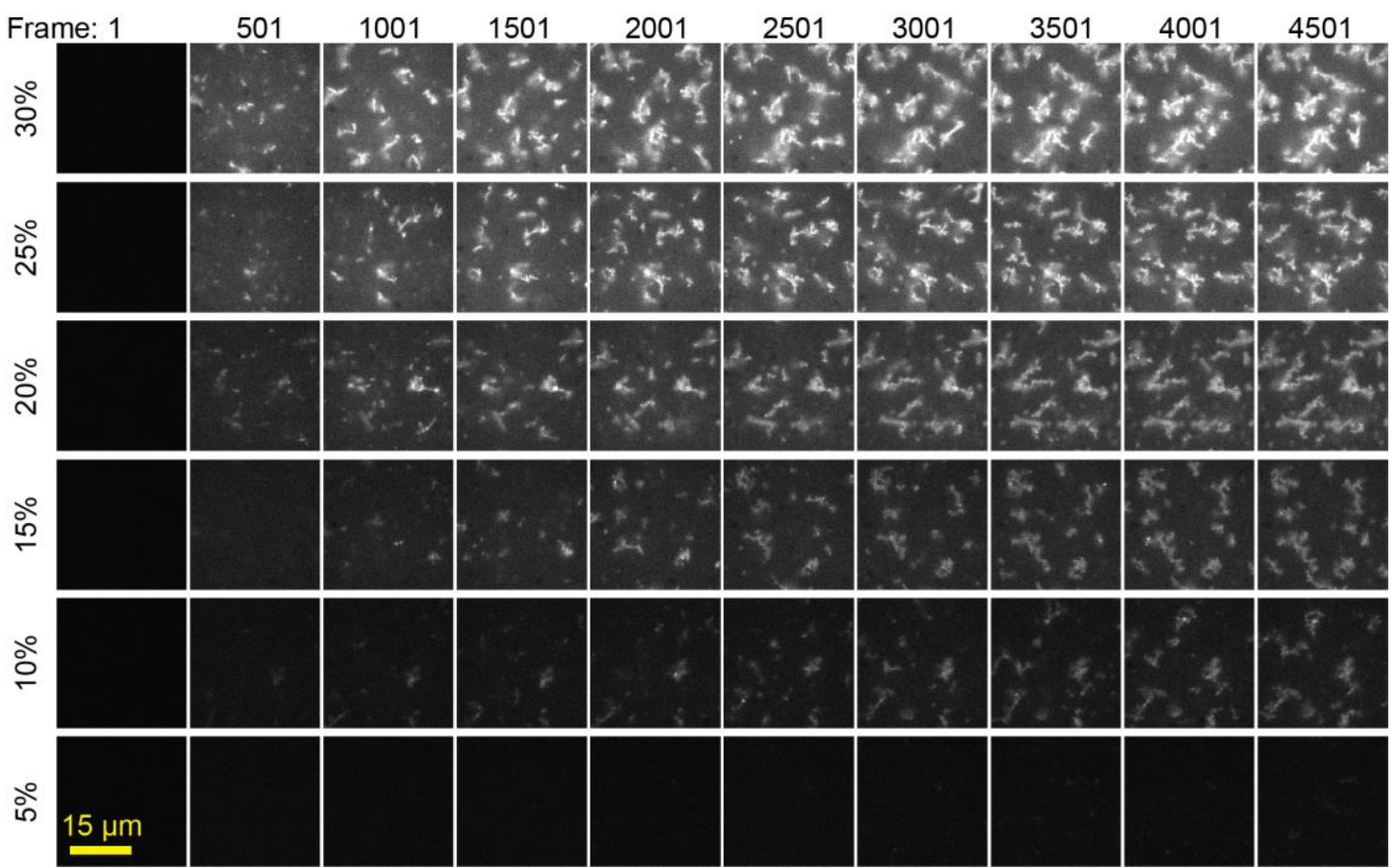

Figure S3. Representative fluorescence images of laser-induced assemblies of AgNPs when illuminated by $405 \mathrm{~nm}$ laser at varying powers $(5 \%-30 \%$, or $\sim 1-6 \mathrm{~mW})$ but a constant initial concentration of AgNPs $(1.3 \mathrm{mg} / \mathrm{mL})$. Scale bar $=15 \mu \mathrm{m}$. 
A

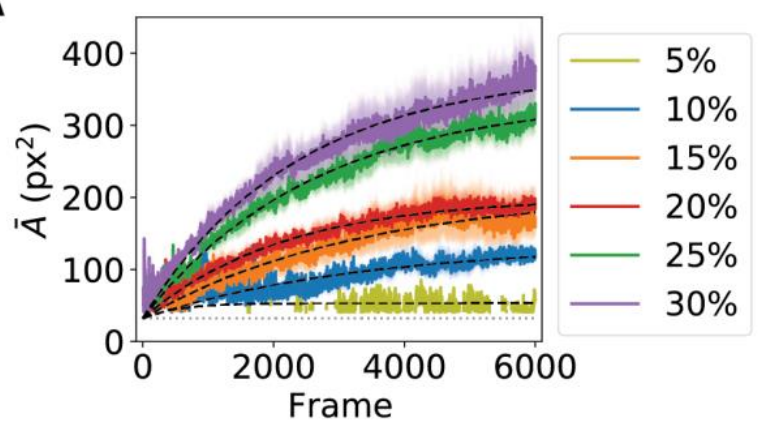

C

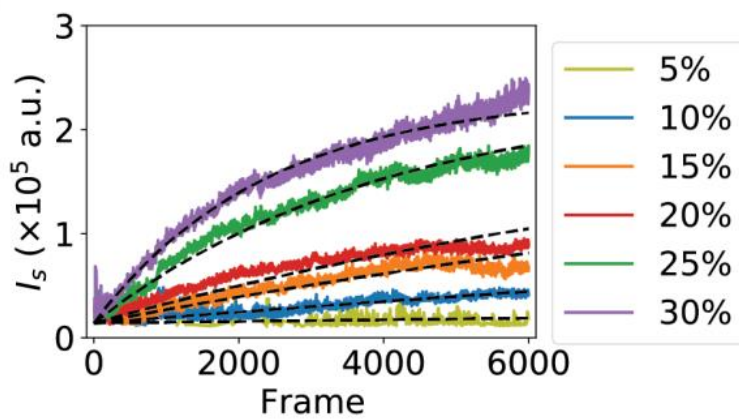

B

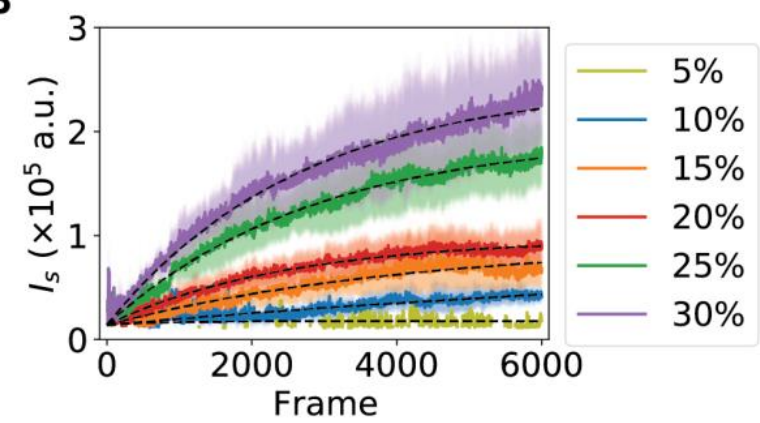

D

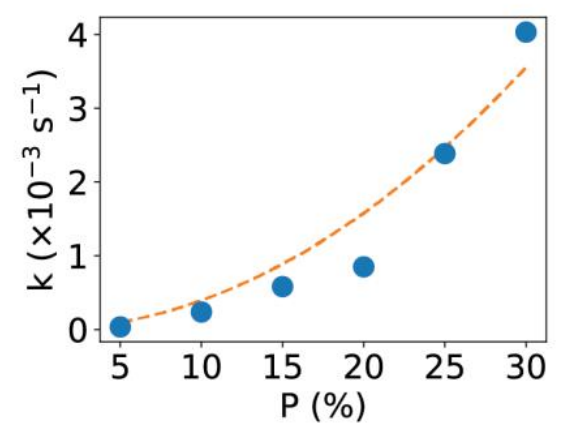

Figure S4. (A) The average sizes of the laser-induced assemblies of AgNPs as functions of time when illuminated with a $405 \mathrm{~nm}$ laser at different power levels (the same data in Fig. 4). The regions of lighter colors indicate the standard errors of the means. The black dashed lines are individually fitted curves using $\bar{A}=A_{0}\left(1-e^{-2 k t}\right)$. (B) The average intensities of the laserinduced assemblies of AgNPs as functions of time when illuminated with a $405 \mathrm{~nm}$ laser at different power levels. The regions of lighter colors indicate the standard errors of the means. The black dashed lines are individually fitted curves using $I_{s}=I_{s 0}\left(1-e^{-2 k t}\right)$. (C) Same data as panel $\mathrm{B}$ but the curves were globally fitted using $I_{s}=I_{s 0}\left(1-e^{-2 k t}\right)$ where $I_{s 0}$ is kept constant for all the curves. (D) Dependence of the fitted growth rate $k$ on the laser power $P$. The orange dashed line is a quadratic fitting. 


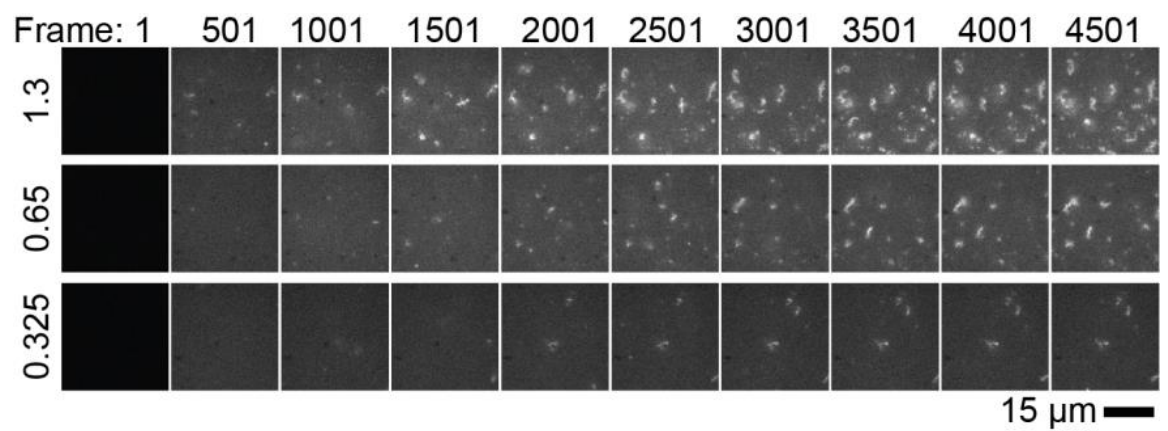

Figure S5. Representative fluorescence images of laser-induced assemblies of AgNPs when illuminated by $405 \mathrm{~nm}$ laser at a constant power $(25 \%$ or $\sim 5 \mathrm{~mW})$ but varying initial concentrations of AgNPs (1.3, 0.65, and $0.325 \mathrm{mg} / \mathrm{mL})$. Scale bar $=15 \mu \mathrm{m}$.

(A)

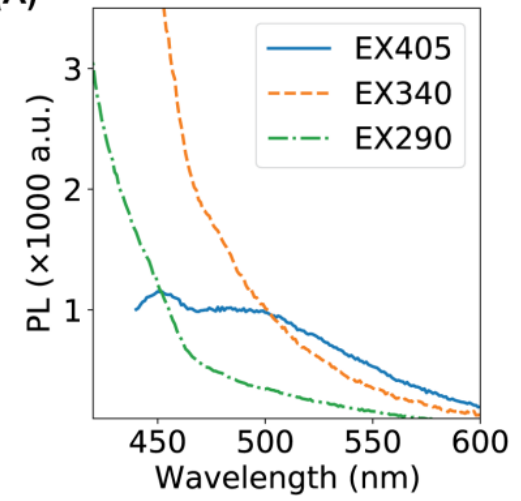

(B)

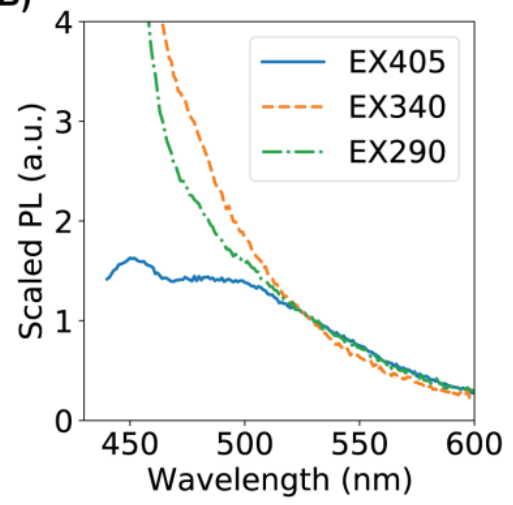

Figure S6. (A) Photoluminescence (PL) spectra of AgNPs at three excitation wavelengths (405 $\mathrm{nm}, 340 \mathrm{~nm}$, and $290 \mathrm{~nm}$ ). (B) Scaled PL spectra of AgNPs (PL = 1.0 at emission wavelength of $530 \mathrm{~nm}$ ) for comparison at the three excitation wavelengths. 


\section{List of Supplementary Movies}

SI Movie M1. A representative movie showing the nanowelding/assembling of AgNPs when illuminated by a $405 \mathrm{~nm}$ laser at $30 \%$ power $(\sim 6 \mathrm{~mW})$. Scale bar $=15 \mu \mathrm{m} .5 \times$ speed-up.

SI Movie M2. An example showing the growth of a small AgNP-assembly and the addition of "monomers". Scale bar $=2 \mu \mathrm{m}$. Real-time.

SI Movie M3. An example showing the merging of assemblies into larger ones. Scale bar $=5$ $\mu \mathrm{m} .5 \times$ speed-up.

SI Movie M4. A representative assembly of AgNPs showing separate fluorescent domains that displayed asynchronous blinking/flashing. Scale bar $=3 \mu \mathrm{m} .5 \times$ speed-up. 